\title{
DECONFINEMENT AND CHIRAL RESTORATION IN NONLOCAL SU(3) CHIRAL QUARK MODELS
}

\author{
J.P. Carlomagno ${ }^{a, b}$, D. Gómez Dumm ${ }^{a, b}$ and N.N. Scoccola ${ }^{b, c, d}$ \\ a IFLP, CONICET - Dpto. de Física, Universidad \\ Nacional de La Plata, C.C. 67, 1900 La Plata, Argentina, \\ ${ }^{b}$ CONICET, Rivadavia 1917, 1033 Buenos Aires, Argentina \\ c Physics Department, Comisión Nacional de Energía Atómica, \\ Av.Libertador 8250, 1429 Buenos Aires, Argentina \\ ${ }^{d}$ Universidad Favaloro, Solís 453, 1078 Buenos Aires, Argentina
}

\begin{abstract}
We study the features of nonlocal SU(3) chiral quark models with wave function renormalization. Model parameters are determined from meson phenomenology, considering different nonlocal form factor shapes. In this context we analyze the characteristics of the deconfinement and chiral restoration transitions at finite temperature, introducing the couplings of fermions to the Polyakov loop. We analyze the results obtained for various thermodynamical quantities considering different Polyakov loop potentials and nonlocal form factors, in comparison with data obtained from lattice QCD calculations.
\end{abstract}

\section{INTRODUCTION}

The detailed understanding of the behavior of strongly interacting matter under extreme conditions of temperature and/or density has become an issue of great interest in recent years. It is widely believed that as the temperature and/or density increase, one finds a transition from a hadronic phase, in which chiral symmetry is broken and quarks are confined, to a partonic phase in which chiral symmetry is restored and/or quarks are deconfined. From the theoretical point of view, one way to address this problem is through lattice QCD calculations [1 $[3]$, which have been significantly improved in the last years. However, this ab initio approach is not yet able to provide a full understanding of the QCD phase diagram and the related hadron properties, owing to the wellknown difficulties of dealing with small current quark masses and finite chemical potentials. Thus, it is worth developing effective models that show consistency with lattice results, and can be extrapolated into regions not accessible by lattice calculation techniques. Here we will concentrate on one particular class of effective theories, namely the so-called nonlocal Polyakov-Nambu-JonaLasinio (nlPNJL) models [4 8], in which quarks move in a background color field and interact through covariant nonlocal chirally symmetric four-point couplings. These approaches, which can 
be considered as an improvement over the (local) PNJL model [9-15], offer a common framework to study both the chiral restoration and deconfinement transitions. In fact, the nonlocal character of the interactions arises naturally in the context of several successful approaches to low-energy quark dynamics [16, 17], and leads to a momentum dependence in the quark propagator that can be made consistent [18] with lattice results [19 21]. Moreover, it has been found that, under certain conditions, it is possible to derive the main features of nlPNJL models starting directly from QCD [22].

Some previous works have addressed the study of nlPNJL models for the case of two dynamical quarks, showing that the presence of nonlocal form factors in the current-current quark interactions leads to a momentum-dependent mass and wave function renormalization (WFR) in the quark propagator 23 25]. As stated, it is possible to choose the model parameters and form factors so as to fit these momentum dependences to those obtained in lattice QCD [18]. The aim of this work is to extend those works to three flavors, including flavor mixing through a nonlocal 't Hooft-like sixfermion interaction. The case of a three-flavor nlPNJL model with simple Gaussian form factors and no WFR in the quark propagator has been previously addressed in Refs. [5], 6], where the phenomenology of light scalar and pseudoscalar mesons is analyzed. In addition, the introduction of a Gaussian form factor to account for the WFR in the three-flavor case has been considered in Ref. [26]. For comparison, we analyze here both the case of a model in which the form factors are Gaussian functions (which ensure a fast ultraviolet convergence of loop integrals), and a model in which these are given by the mentioned lattice QCD-inspired functions [see Eqs. (29)30) below] of the momentum. In this framework we determine several properties of light mesons (masses, mixing angles, decay constants), analyzing the compatibility with the corresponding phenomenological values. Then we study the deconfinement and chiral restoration phase transitions that occur at finite temperature, and we determine the corresponding critical temperatures. Our analyses are carried out at the mean field level, considering the above-mentioned form factor shapes and various parameter sets and functional forms for the Polyakov potential. We also analyze the behavior of thermodynamical quantities such as the interaction energy and the entropy and energy densities. The results are discussed in comparison with data obtained from lattice QCD calculations.

This article is organized as follows. In Sec. II we present the general formalism, including analytical results for the scalar and pseudoscalar meson properties. We discuss the model parameterization and compare our predictions with phenomenological expectations. In Sec. III we extend our analysis to nonzero temperature. The Polyakov loop potential is introduced and the deconfinement and chiral restoration phase transitions are analyzed. In Sec. IV we summarize our 
results and conclusions. The Appendix includes some of our analytical expressions.

\section{NONLOCAL SU(3) CHIRAL QUARK MODEL - ZERO TEMPERATURE}

We start by considering the Euclidean effective action

$$
\begin{aligned}
S_{E}= & \int d^{4} x\left\{\bar{\psi}(x)(-\imath \not D+\hat{m}) \psi(x)-\frac{G}{2}\left[j_{a}^{S}(x) j_{a}^{S}(x)+j_{a}^{P}(x) j_{a}^{P}(x)+j^{r}(x) j^{r}(x)\right]\right. \\
& \left.-\frac{H}{4} A_{a b c}\left[j_{a}^{S}(x) j_{b}^{S}(x) j_{c}^{S}(x)-3 j_{a}^{S}(x) j_{b}^{P}(x) j_{c}^{P}(x)\right]+\mathcal{U}[\mathcal{A}(x)]\right\},
\end{aligned}
$$

where $\psi(x)$ is the $N_{f}=3$ fermion triplet $\psi=(u d s)^{T}$, and $\hat{m}=\operatorname{diag}\left(m_{u}, m_{d}, m_{s}\right)$ is the current quark mass matrix. We will work in the isospin symmetry limit, assuming $m_{u}=m_{d}$. The fermion currents are given by

$$
\begin{aligned}
& j_{a}^{s}(x)=\int d^{4} z g(z) \bar{\psi}\left(x+\frac{z}{2}\right) \lambda_{a} \psi\left(x-\frac{z}{2}\right), \\
& j_{a}^{p}(x)=\int d^{4} z g(z) \bar{\psi}\left(x+\frac{z}{2}\right) \imath \lambda_{a} \gamma_{5} \psi\left(x-\frac{z}{2}\right), \\
& j^{r}(x)=\int d^{4} z f(z) \bar{\psi}\left(x+\frac{z}{2}\right) \frac{\imath \overleftrightarrow{\partial}}{2 \kappa} \psi\left(x-\frac{z}{2}\right),
\end{aligned}
$$

where $f(z)$ and $g(z)$ are covariant form factors responsible for the nonlocal character of the interactions, and $\lambda_{a}, a=0, \ldots, 8$, are the standard eight Gell-Mann matrices, plus $\lambda_{0}=\sqrt{2 / 3} \mathbf{1}_{3 \times 3}$. The relative weight of the interaction driven by $j^{r}(x)$, which is responsible for the quark wave function renormalization, is controlled by the parameter $\kappa$. The model includes flavor mixing through a 't Hooft-like term, in which the $\mathrm{SU}(3)$ symmetric constants $A_{a b c}$ are defined by

$$
A_{a b c}=\frac{1}{3 !} \epsilon_{i j k} \epsilon_{m n l}\left(\lambda_{a}\right)_{i m}\left(\lambda_{b}\right)_{j n}\left(\lambda_{c}\right)_{k l} .
$$

The interaction between fermions and color gauge fields $G_{\mu}^{a}$ takes place through the covariant derivative in the fermion kinetic term, $D_{\mu} \equiv \partial_{\mu}-\imath \mathcal{A}_{\mu}$, where $\mathcal{A}_{\mu}=g G_{\mu}^{a} \lambda^{a} / 2$. Finally, the action includes an effective potential $\mathcal{U}$ that accounts for gauge field self-interactions. At the mean field level we will assume that fermions move on a uniform background gauge field, which for zero temperature decouples from matter (finite temperature effects will be discussed in the next sections).

To work with mesonic degrees of freedom we proceed to perform a standard bosonization of the fermionic theory, introducing scalar fields $\sigma_{a}(x), \zeta(x)$ and pseudoscalar fields $\pi_{a}(x)$, together with auxiliary fields $S_{a}(x), P_{a}(x)$ and $R(x)$, with $a=0, \ldots, 8$. After integrating out the fermion fields 
we obtain a partition function

$$
\begin{aligned}
\mathcal{Z} & =\int \mathcal{D} \sigma_{a} \mathcal{D} \pi_{a} \mathcal{D} \zeta A\left(\sigma_{a}, \pi_{a}, \zeta\right) \\
& \times \int \mathcal{D} S_{a} \mathcal{D} P_{a} \mathcal{D} R \exp \int d^{4} x\left[\sigma_{a} S_{a}+\pi_{a} P_{a}+\zeta R+\frac{G}{2}\left(S_{a} S_{a}+P_{a} P_{a}+R^{2}\right)+\right. \\
& \left.+\frac{H}{4} A_{a b c}\left(S_{a} S_{b} S_{c}-3 S_{a} P_{b} P_{c}\right)\right]
\end{aligned}
$$

where the operator $A\left(p, p^{\prime}\right)$ (in momentum space) is given by

$$
\begin{aligned}
A\left(p, p^{\prime}\right) & =(2 \pi)^{4} \delta^{(4)}\left(p-p^{\prime}\right)\left(-\not p+m_{c}\right)+g\left(\frac{p+p^{\prime}}{2}\right)\left[\sigma_{a}\left(p^{\prime}-p\right)+\imath \gamma_{5} \pi_{a}\left(p^{\prime}-p\right)\right] \lambda_{a}+ \\
& +\frac{1}{2 \kappa} f\left(\frac{p+p^{\prime}}{2}\right)\left(\not p+\not p^{\prime}\right) \zeta\left(p^{\prime}-p\right) .
\end{aligned}
$$

Now we follow the stationary phase approximation, replacing the path integrals over the auxiliary fields by the corresponding argument evaluated at the minimizing values $\tilde{S}_{a}, \tilde{P}_{a}$, and $\tilde{R}$. The procedure is similar to that carried out in Ref. [27], where more details can be found.

\section{A. Mean Field Approximation}

We consider the mean field approximation (MFA), in which the meson fields are expanded around their vacuum expectation values. One thus has

$$
\begin{aligned}
\sigma_{a}(x) & =\bar{\sigma}_{a}+\delta \sigma_{a}(x), \\
\pi_{a}(x) & =\delta \pi_{a}(x) \\
\zeta(x) & =\bar{\zeta}+\delta \zeta(x),
\end{aligned}
$$

where we have assumed that pseudoscalar mean field values vanish, owing to parity conservation. Moreover, for the scalar fields only $\bar{\sigma}_{0,8}$ and $\bar{\zeta}$ can be different from zero due to charge and isospin symmetries. Thus, the Euclidean action reduces to

$$
\frac{S_{E}^{\mathrm{MFA}}}{V^{(4)}}=-2 \operatorname{Tr} \int \frac{d^{4} p}{(2 \pi)^{4}} \log \left[\frac{M^{2}(p)+p^{2}}{Z^{2}(p)}\right]-\bar{\sigma}_{a} \bar{S}_{a}-\bar{\zeta} \bar{R}-\frac{G}{2}\left(\bar{S}_{a} \bar{S}_{a}+\bar{R}^{2}\right)-\frac{H}{4} A_{a b c} \bar{S}_{a} \bar{S}_{b} \bar{S}_{c},
$$

where $\bar{S}_{a}, \bar{P}_{a}$, and $\bar{R}$ stand for the values of $\tilde{S}_{a}, \tilde{P}_{a}$ and $\tilde{R}$ within the MFA.

For the neutral fields $(a=0,3,8)$ it is convenient to change to a flavour basis, $\phi_{a} \rightarrow \phi_{i}$, where $i=u, d, s$, or equivalently $i=1,2,3$. In this basis, by minimizing the mean field action in Eq. (7) 
we obtain the gap equations given in Ref. [27],

$$
\begin{gathered}
\bar{\sigma}_{u}+G \bar{S}_{u}+\frac{H}{2} \bar{S}_{d} \bar{S}_{s}=0, \\
\bar{\sigma}_{d}+G \bar{S}_{d}+\frac{H}{2} \bar{S}_{s} \bar{S}_{u}=0, \\
\bar{\sigma}_{s}+G \bar{S}_{s}+\frac{H}{2} \bar{S}_{u} \bar{S}_{d}=0,
\end{gathered}
$$

plus an extra equation arising from the $j^{r}(x)$ current-current interaction,

$$
\bar{\zeta}+G \bar{R}=0
$$

where the mean field values $\bar{S}_{i}$ and $\bar{R}$ are given by

$$
\begin{aligned}
\bar{S}_{i} & =-8 N_{c} \int \frac{d^{4} p}{(2 \pi)^{4}} g(p) \frac{Z(p) M_{i}(p)}{p^{2}+M_{i}^{2}(p)}, i=u, d, s, \\
\bar{R} & =\frac{4 N_{c}}{\kappa} \int \frac{d^{4} p}{(2 \pi)^{4}} p^{2} f(p) \sum_{i=1}^{3} \frac{Z(p)}{p^{2}+M_{i}^{2}(p)} .
\end{aligned}
$$

The functions $M_{i}(p)$ and $Z(p)$ correspond to momentum-dependent effective masses and WFR of the quark propagators. In terms of the model parameters and form factors, these are given by

$$
\begin{aligned}
M_{i}(p) & =Z(p)\left[m_{i}+\bar{\sigma}_{i} g(p)\right], \\
Z(p) & =\left[1-\frac{\bar{\zeta}}{\kappa} f(p)\right]^{-1}
\end{aligned}
$$

Thus, for a given set of model parameters and form factors, from Eqs. (8) 11) one can numerically obtain the mean field values $\bar{\sigma}_{u, s}$ and $\bar{\zeta}$.

The chiral condensates $\langle\bar{q} q\rangle$, order parameters of the chiral restoration transition, can be obtained by varying the MFA partition function with respect to the current quark masses. These quantities are, in general, divergent and can be regularized by subtracting the free quark contributions. One has

$$
\langle\bar{q} q\rangle=-4 N_{c} \int \frac{d^{4} p}{(2 \pi)^{4}}\left[\frac{Z(p) M_{q}(p)}{p^{2}+M_{q}^{2}(p)}-\frac{m_{q}}{p^{2}+m_{q}^{2}}\right], q=u, d, s .
$$

\section{B. Quadratic Fluctuations - Meson masses and weak decay constants}

In order to analyze the properties of meson fields it is necessary to go beyond the MFA, considering quadratic fluctuations in the Euclidean action:

$$
S_{E}^{\text {quad }}=\frac{1}{2} \int \frac{d^{4} p}{(2 \pi)^{4}} \sum_{M} r_{M} G_{M}\left(p^{2}\right) \phi_{M}(p) \bar{\phi}_{M}(-p)
$$


where meson fluctuations $\delta \sigma_{a}, \delta \pi_{a}$ have been translated to a charge basis $\phi_{M}, M$ being the scalar and pseudoscalar mesons in the lowest mass nonets $\left(\sigma, \pi^{0}\right.$, etc.), plus the $\zeta$ field. The coefficient $r_{M}$ is 1 for charge eigenstates $M=\mathrm{a}_{0}^{0}, \sigma, f_{0}, \zeta, \pi^{0}, \eta, \eta^{\prime}$, and 2 for $M=\mathrm{a}_{0}^{+}, K_{0}^{*+}, K_{0}^{* 0}, \pi^{+}, K^{+}, K^{0}$. Meson masses are then given by the equations

$$
G_{M}\left(-m_{M}^{2}\right)=0
$$

In addition, physical states have to be normalized through

$$
\tilde{\phi}_{M}(p)=Z_{M}^{-1 / 2} \phi_{M}(p)
$$

where

$$
Z_{M}^{-1}=\left.\frac{d G_{M}(p)}{d p^{2}}\right|_{p^{2}=-m_{M}^{2}} .
$$

The full expressions for the one-loop functions $G_{M}(q)$ are quoted in the Appendix. They can be written in terms of the coupling constants $G$ and $H$, the mean field values $\bar{S}_{u, s}$, and quark loop functions that prove to be ultraviolet convergent, owing to the asymptotic behavior of the nonlocal form factors. For the pseudoscalar meson sector, the $\pi$ and $K$ mesons decouple, while the $I=0$ states get mixed. Since the corresponding mixing angles are momentum-dependent functions, it is necessary to introduce two mixing angles $\theta_{\eta}$ and $\theta_{\eta^{\prime}}$, defined at $p^{2}=-m_{\eta}^{2}$ and $p^{2}=-m_{\eta^{\prime}}^{2}$ respectively, see Eq. (49). In the case of the scalar meson sector, the $\mathrm{a}_{0}$ and $K_{0}^{*}$ mesons decouple, while the $\zeta, \sigma_{0}$, and $\sigma_{8}$ fields get mixed by a $3 \times 3$ matrix, see Eq. (50).

One can also calculate the weak decay constants of pseudoscalar mesons. These are given by the matrix elements of the axial currents $A_{\mu}^{a}$ between the vacuum and the physical meson states,

$$
\imath f_{a b}\left(p^{2}\right) p_{\mu}=\left\langle 0\left|A_{\mu}^{a}(0)\right| \delta \pi_{b}(p)\right\rangle .
$$

The matrix elements can be calculated from the expansion of the Euclidean effective action in the presence of external axial currents,

$$
\left\langle 0\left|A_{\mu}^{a}(0)\right| \delta \pi_{b}(p)\right\rangle=\left.\frac{\delta^{2} S_{E}}{\delta A_{\mu}^{a} \delta \pi_{b}(p)}\right|_{A_{\mu}^{a}=\delta \pi_{b}=0} .
$$

It is important to notice that, owing to nonlocality, the axial currents have to be introduced not only into the covariant derivative in the Euclidean action, but also in the fermion fields entering the nonlocal currents, through the replacements [27, 28]

$$
\begin{gathered}
\psi\left(x-\frac{z}{2}\right) \longrightarrow W_{A}\left(x, x-\frac{z}{2}\right) \psi\left(x-\frac{z}{2}\right) \\
\psi^{\dagger}\left(x+\frac{z}{2}\right) \longrightarrow \psi^{\dagger}\left(x+\frac{z}{2}\right) W_{A}\left(x+\frac{z}{2}, x\right) .
\end{gathered}
$$


Here the transport function $W_{A}(x, y)$ is given by

$$
W_{A}(x, y)=P \exp \left\{\frac{\imath}{2} \int_{x}^{y} d s_{\mu} \gamma_{5} \lambda_{a} A_{\mu}^{a}(s)\right\},
$$

where $s$ runs over an arbitrary path connecting $x$ with $y$.

After a rather lengthy calculation, we find that the relevant term in the expansion of the Euclidean action can be written as

$$
S_{E}^{[A, \phi]}=\int \frac{d^{4} p}{(2 \pi)^{4}} \frac{d^{4} p^{\prime}}{(2 \pi)^{4}} \sum_{i, j=1}^{3} A_{\mu i j}(p) \delta \pi_{j i}\left(p^{\prime}\right) G_{i j}^{\mu}\left(p, p^{\prime}\right),
$$

where we have defined $A_{\mu}=\lambda_{a} A_{\mu}^{a} / \sqrt{2}, \delta \pi=\lambda_{a} \delta \pi_{a} / \sqrt{2}$. The functions $G_{i j}^{\mu}\left(p, p^{\prime}\right)$ are found to satisfy the relation

$$
p_{\mu} G_{i j}^{\mu}\left(p, p^{\prime}\right)=-i \delta^{(4)}\left(p+p^{\prime}\right) F_{i j}\left(p^{2}\right)
$$

where

$$
\begin{aligned}
F_{i j}\left(p^{2}\right)= & 2 N_{c} \int \frac{d^{4} q}{(2 \pi)^{4}}\left[g\left(q^{+}\right)+g\left(q^{-}\right)-2 g(q)\right] Z(q)\left[\frac{M_{i}(q)}{q^{2}+M_{i}^{2}(q)}+\frac{M_{j}(q)}{q^{2}+M_{j}^{2}(q)}\right] \\
& -2 N_{c} \int \frac{d^{4} q}{(2 \pi)^{4}}\left(\bar{\sigma}_{i}+\bar{\sigma}_{j}\right)\left[g\left(q^{+}\right)+g\left(q^{-}\right)-2 g(q)\right] g(q) \\
& \times \frac{Z\left(q^{+}\right)}{M_{i}^{2}\left(q^{+}\right)+q^{+2}} \frac{Z\left(q^{-}\right)}{M_{j}^{2}\left(q^{-}\right)+q^{-2}}\left[\left(q^{+} \cdot q^{-}\right)+M_{i}\left(q^{+}\right) M_{j}\left(q^{-}\right)\right] \\
& +4 N_{c} \int \frac{d^{4} q}{(2 \pi)^{4}} g(q) \frac{\left[M_{i}\left(q^{+}\right) q^{-}-M_{j}\left(q^{-}\right) q^{+}\right] \cdot\left[Z\left(q^{-}\right) q^{+}-Z\left(q^{+}\right) q^{-}\right]}{\left[q^{+2}+M_{i}^{2}\left(q^{+}\right)\right]\left[q^{-2}+M_{j}^{2}\left(q^{-}\right)\right]}
\end{aligned}
$$

with $q^{ \pm}=q \pm p / 2$. It is worth pointing out that the functions $F_{i j}$ (and, therefore, the weak decay constants) are given by the longitudinal component of $G_{i j}^{\mu}\left(p, p^{\prime}\right)$, which does not depend on the arbitrary path chosen in the transport functions $W_{A}(x, y)$.

From the above expressions, the weak decay constants for $\pi$ and $K$ mesons in the isospin limit are given by

$$
\begin{aligned}
& f_{\pi}=\left.\frac{Z_{\pi}^{1 / 2}}{m_{\pi}^{2}} F_{u u}\left(p^{2}\right)\right|_{p^{2}=-m_{\pi}^{2}}, \\
& f_{K}=\left.\frac{Z_{K}^{1 / 2}}{m_{K}^{2}} F_{u s}\left(p^{2}\right)\right|_{p^{2}=-m_{K}^{2}} .
\end{aligned}
$$

For the $\eta-\eta^{\prime}$ sector, the functions $f_{a b}\left(p^{2}\right)$ defined in Eq. (17) are related to $F_{i j}\left(p^{2}\right)$ through

$$
\begin{aligned}
& f_{00}\left(p^{2}\right)=\frac{1}{3}\left[2 F_{u u}\left(p^{2}\right)+F_{s s}\left(p^{2}\right)\right], \\
& f_{88}\left(p^{2}\right)=\frac{1}{3}\left[F_{u u}\left(p^{2}\right)+2 F_{s s}\left(p^{2}\right)\right], \\
& f_{08}\left(p^{2}\right)=\frac{\sqrt{2}}{3}\left[F_{u u}\left(p^{2}\right)-F_{s s}\left(p^{2}\right)\right] .
\end{aligned}
$$


These can be translated to the mass eigenstate basis through the mixing angles in Eq. (49). Thus one defines

$$
\begin{aligned}
f_{\eta}^{a} & =\left.\frac{Z_{\eta}^{1 / 2}}{m_{\eta}^{2}}\left[f_{a 8}\left(p^{2}\right) \cos \theta_{\eta}-f_{a 0}\left(p^{2}\right) \sin \theta_{\eta}\right]\right|_{p^{2}=-m_{\eta}^{2}}, \quad a=0,8, \\
f_{\eta^{\prime}}^{a} & =\left.\frac{Z_{\eta^{\prime}}^{1 / 2}}{m_{\eta^{\prime}}^{2}}\left[f_{a 8}\left(p^{2}\right) \sin \theta_{\eta^{\prime}}+f_{a 0}\left(p^{2}\right) \cos \theta_{\eta^{\prime}}\right]\right|_{p^{2}=-m_{\eta^{\prime}}^{2}}, \quad a=0,8 .
\end{aligned}
$$

In order to compare with phenomenological determinations, it is convenient to consider an alternative parametrization in terms of two decay constants $f_{0}, f_{8}$ and two mixing angles $\theta_{0}, \theta_{8}$ [29, 30]. Both parametrizations are related by

$$
\left(\begin{array}{cc}
f_{\eta}^{8} & f_{\eta}^{0} \\
f_{\eta^{\prime}}^{8} & f_{\eta^{\prime}}^{0}
\end{array}\right)=\left(\begin{array}{cc}
f_{8} \cos \theta_{8} & -f_{0} \sin \theta_{0} \\
f_{8} \sin \theta_{8} & f_{0} \cos \theta_{0}
\end{array}\right)
$$

\section{Model parameters And form factors}

The model includes five parameters, namely the current quark masses $m_{u, s}$ and the coupling constants $G, H$, and $\kappa$. In addition, one has to specify the form factors $f(z)$ and $g(z)$ entering the nonlocal fermion currents. Here, following Ref. [18], we will consider two parameter sets, corresponding to two different functional forms for $f(z)$ and $g(z)$. The first one corresponds to the often-used exponential forms

$$
g(p)=\exp \left(-p^{2} / \Lambda_{0}^{2}\right), \quad f(p)=\exp \left(-p^{2} / \Lambda_{1}^{2}\right)
$$

which guarantee a fast ultraviolet convergence of the loop integrals. Note that the range (in momentum space) of the nonlocality in each channel is determined by the parameters $\Lambda_{0}$ and $\Lambda_{1}$, respectively. The second set of form factors considered here is

$$
g(p)=\frac{1+\alpha_{z}}{1+\alpha_{z} f_{z}(p)} \frac{\alpha_{m} f_{m}(p)-m \alpha_{z} f_{z}(p)}{\alpha_{m}-m \alpha_{z}}, \quad f(p)=\frac{1+\alpha_{z}}{1+\alpha_{z} f_{z}(p)} f_{z}(p),
$$

where

$$
f_{m}(p)=\left[1+\left(p^{2} / \Lambda_{0}^{2}\right)^{3 / 2}\right]^{-1}, \quad f_{z}(p)=\left[1+\left(p^{2} / \Lambda_{1}^{2}\right)\right]^{-5 / 2} .
$$

As shown in Ref. [18], for the $\mathrm{SU}(2)$ version of the model these functional forms can very well reproduce the momentum dependence of mass and wave function renormalization obtained in lattice calculations.

Given the form factor functions, one can fix the model parameters so as to reproduce the observed meson phenomenology. To the above-mentioned parameters $m_{u, s}, G, H$, and $\kappa$ one has 
to add the cutoffs $\Lambda_{0}$ and $\Lambda_{1}$, introduced through the form factors. Here we have chosen to take as input value the light quark mass $m_{u}$, while the remaining six parameters are determined by fixing the value of the quark WFR at momentum zero, $Z(0)=0.7$ (as dictated by lattice QCD estimations), and by requiring that the model reproduces the empirical values of five physical quantities. These are the masses of the pseudoscalar mesons $\pi, K$ and $\eta^{\prime}$, the pion weak decay constant $f_{\pi}$ and the light quark condensate $\langle\bar{u} u\rangle$. In Table I we quote the numerical results for the model parameters that we have obtained for the above-described form factor functions. In what follows, the parameter sets corresponding to the form factors in Eqs. (28) and (29, 30) will be referred to as set I and set II, respectively. As expected from the ansatz chosen for the form factors, for set II the momentum-dependent mass and WFR in the light quark propagators are able to fit adequately the results obtained in lattice QCD calculations. This is shown in Fig. 1, where we plot the curves obtained for the functions $M(p)$ and $Z(p)$, together with $N_{f}=2+1$ lattice data taken from Ref. [20]. For comparison we also quote the results corresponding to set I.

\begin{tabular}{ccc}
\hline \hline & Set I & Set II \\
\hline \hline$m_{u}[\mathrm{MeV}]$ & 5.7 & 2.5 \\
$m_{s}[\mathrm{MeV}]$ & 136 & 63.9 \\
$G \Lambda_{0}^{2}$ & 23.64 & 15.55 \\
$-H \Lambda_{0}^{5}$ & 526 & 241 \\
$\kappa[\mathrm{GeV}]$ & 4.36 & 8.08 \\
$\Lambda_{0}[\mathrm{GeV}]$ & 0.814 & 0.824 \\
$\Lambda_{1}[\mathrm{GeV}]$ & 1.032 & 1.550 \\
\hline
\end{tabular}

Table I: Model parameters for the form factors in Eqs. (28) (set I) and (29) (s0) (set II).

\section{Meson phenomenology}

Once the parameters have been determined, we can calculate the values of several meson properties for the scalar and pseudoscalar sectors. Our numerical results for sets I and II are summarized in Table II, together with the corresponding phenomenological estimates. The quantities marked with an asterisk are those that have been chosen as input values. In general, it is seen that the meson masses, mixing angles, and weak decay constants predicted by the model are in reasonable agreement with phenomenological expectations. Moreover, the results for set I do not differ significantly from those obtained in Ref. [6] for a nIPNJL model with a Gaussian form factor $g(p)$ and 

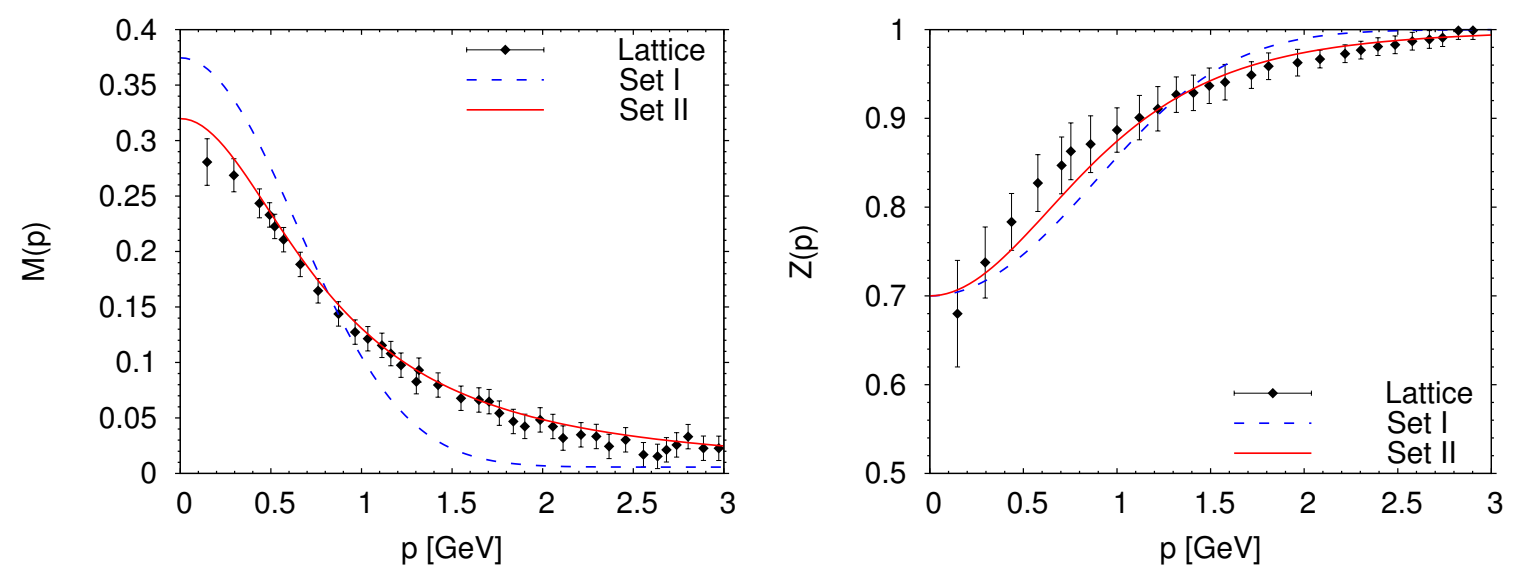

Figure 1: Mass and WFR as functions of the momentum for our parameterizations sets I and II, in comparison with lattice results from Ref. [20].

no WFR. Regarding the scalar meson sector, a new ingredient with respect to the model with no WFR is the presence of the additional field $\zeta$, which mixes with the $I=0$ fields $\sigma_{0}$ and $\sigma_{8}$. The mass of the physical particles can be obtained by determining the zeros of the functions $G_{\zeta, \sigma, f_{0}}\left(p^{2}\right)$ arising from the diagonalization of the $3 \times 3$ matrix in Eq. (50) (see the Appendix). From the corresponding numerical calculation it is seen that one of these functions is positive definite for the momentum range described by our models, which reflects that the eigenstate associated with $\zeta$ does not correspond to a physical particle. For the remaining two states, which can be interpreted as the $f_{0}(500)$ (or $\sigma$ ) and $f_{0}(980)$ scalar mesons quoted by the Particle Data Group (PDG) [31], we obtain masses of about 550 and $1200 \mathrm{MeV}$. In fact, in the case of the $f_{0}$ meson it happens that the loop integrals in Eq. (51) become divergent, and need some regularization prescription. This occurs since $p^{2}$ exceeds a threshold above which both effective quarks can be simultaneously on shell, which can be interpreted as the possibility of a decay of the meson into two massive quarks. The integrals can be properly defined e.g. following the prescription in Ref. [27]. Since the threshold lies at about $1 \mathrm{GeV}$, we have estimated the mass value for the $f_{0}$ meson by an extrapolation from the momentum region in which the integrals are well defined. Following a similar procedure, the masses of $K_{0}^{*}$ charged and neutral mesons are found to be $\simeq 1300 \mathrm{MeV}$; thus, these particles can be identified with the $K_{0}^{*}(1430)$ mesons quoted by the PDG $\left(m_{K_{0}^{*}}=1425 \pm 50 \mathrm{MeV}\right)$ [31]. Our models do not seem to include the light strange scalar mesons $\kappa$, which indeed still need confirmation and thus have been omitted from PDG particle summary tables. 


\begin{tabular}{|c|c|c|c|}
\hline & Set I & Set II & Empirical \\
\hline $\bar{\sigma}_{u}[\mathrm{MeV}]$ & 529 & 454 & - \\
\hline $\bar{\sigma}_{s}[\mathrm{MeV}]$ & 702 & 663 & - \\
\hline $\bar{\zeta} / \kappa$ & -0.429 & -0.429 & - \\
\hline$-\langle\bar{u} u\rangle^{1 / 3}[\mathrm{MeV}]^{*}$ & 240 & 320 & - \\
\hline$-\langle\bar{s} s\rangle^{1 / 3}[\mathrm{MeV}]$ & 198 & 343 & - \\
\hline$m_{\pi}[\mathrm{MeV}] *$ & 139 & 139 & 139 \\
\hline$m_{K}[\mathrm{MeV}] *$ & 495 & 495 & 495 \\
\hline$m_{\eta}[\mathrm{MeV}]$ & 527 & 537 & 547 \\
\hline$m_{\eta^{\prime}}[\mathrm{MeV}]^{*}$ & 958 & 958 & 958 \\
\hline$m_{\mathrm{a}_{0}}[\mathrm{MeV}]$ & 936 & 916 & 980 \\
\hline$m_{K_{0}^{*}}[\mathrm{MeV}]$ & 1300 & 1300 & 1425 \\
\hline$m_{\sigma}[\mathrm{MeV}]$ & 599 & 537 & $400-550$ \\
\hline$m_{f_{0}}[\mathrm{MeV}]$ & 1300 & 1200 & 990 \\
\hline$f_{\pi}[\mathrm{MeV}] *$ & 92.4 & 92.4 & 92.4 \\
\hline$f_{K} / f_{\pi}$ & 1.17 & 1.16 & 1.22 \\
\hline$f_{\eta}^{0} / f_{\pi}$ & 0.17 & 0.14 & $(0.11-0.507)$ \\
\hline$f_{\eta}^{8} / f_{\pi}$ & 1.12 & 1.12 & $(1.17-1.22)$ \\
\hline$f_{\eta^{\prime}}^{0} / f_{\pi}$ & 1.09 & 1.43 & $(0.98-1.16)$ \\
\hline$f_{\eta^{\prime}}^{8} / f_{\pi}$ & -0.48 & -0.42 & $-(0.42-0.46)$ \\
\hline$\theta_{\eta}$ & $-2.95^{\circ}$ & $-1.01^{\circ}$ & - \\
\hline$\theta_{\eta^{\prime}}$ & $-41.62^{\circ}$ & $-30.79^{\circ}$ & - \\
\hline$\theta_{0}$ & $-8.63^{\circ}$ & $-5.53^{\circ}$ & $-\left(0^{\circ}-10^{\circ}\right)$ \\
\hline$\theta_{8}$ & $-22.94^{\circ}$ & $-20.67^{\circ}$ & $-\left(19^{\circ}-22^{\circ}\right)$ \\
\hline
\end{tabular}

Table II: Numerical results for various phenomenological quantities. Input values are marked with an asterisk.

Concerning the quark masses and condensates, it is found that in the case of set II we obtain relatively low values for $m_{u}$ and $m_{s}$, and a somewhat large value for the light quark condensate. Similar results have previously been obtained in Refs. [18] and [26], within two- and three-flavor parameterizations respectively. As discussed in those articles, this can be in part attributed to the fact that our fit to lattice data for the function $Z(p)$ is based on the calculations in Ref. [20], which correspond to a rather large renormalization scale $\mu=3 \mathrm{GeV}$. On the other hand, for both sets I and II we find that the quark mass ratio is $m_{s} / m_{u} \simeq 25$, which is phenomenologically adequate. Something similar happens with the product $-\langle\bar{u} u\rangle m_{u}$, which gives $7.9 \times 10^{-5} \mathrm{GeV}^{4}$ for set I and 
$8.2 \times 10^{-5} \mathrm{GeV}^{4}$ for set II: these values are in agreement with the scale-independent result obtained from the Gell-Mann-Oakes-Renner relation at the leading order in the chiral expansion, namely $-\langle\bar{u} u\rangle m_{u}=f_{\pi}^{2} m_{\pi}^{2} / 2 \simeq 8.3 \times 10^{-5} \mathrm{GeV}^{4}$.

\section{NONZERO TEMPERATURE}

\section{A. Polyakov loop}

As stated in the previous section, the effective action of the model includes the interaction of quarks with color gauge fields through the covariant derivative in the fermion kinetic term. This coupling will be treated at the mean field level, considering that quarks move on a constant background field $\phi=A_{4}=i A_{0}=i g \delta_{\mu 0} G_{a}^{\mu} \lambda^{a} / 2$, where $G_{a}^{\mu}$ are the $\mathrm{SU}(3)$ color gauge fields. Then the traced Polyakov loop, which in the infinite quark mass limit can be taken as order parameter of confinement, is given by $\Phi=\frac{1}{3} \operatorname{Tr} \exp (i \phi / T)$. We will work in the so-called Polyakov gauge, in which the matrix $\phi$ is given a diagonal representation $\phi=\phi_{3} \lambda_{3}+\phi_{8} \lambda_{8}$. Owing to the charge conjugation properties of the QCD Lagrangian [32], the mean field traced Polyakov loop field $\Phi$ is expected to be a real quantity. Assuming that $\phi_{3}$ and $\phi_{8}$ are real valued [13], this implies $\phi_{8}=0$, $\Phi=\left[2 \cos \left(\phi_{3} / T\right)+1\right] / 3$.

The effective gauge field self-interactions are given by the Polyakov-loop potential $\mathcal{U}[A(x)]$. At finite temperature $T$, it is normal to take for this potential a functional form based on properties of pure gauge QCD. One possible ansatz is that based on the logarithmic expression of the Haar measure associated with the $\mathrm{SU}(3)$ color group integration. The corresponding potential is given by [13]

$$
\frac{\mathcal{U}_{\log }(\Phi, T)}{T^{4}}=-\frac{1}{2} a(T) \Phi^{2}+b(T) \log \left(1-6 \Phi^{2}+8 \Phi^{3}-3 \Phi^{4}\right)
$$

where

$$
a(T)=a_{0}+a_{1}\left(\frac{T_{0}}{T}\right)+a_{2}\left(\frac{T_{0}}{T}\right)^{2}, \quad b(T)=b_{3}\left(\frac{T_{0}}{T}\right)^{3} .
$$

The parameters can be fitted to pure gauge lattice QCD data so as to properly reproduce the corresponding equation of state and Polyakov loop behavior. This leads to [13]

$$
a_{0}=3.51, \quad a_{1}=-2.47, \quad a_{2}=15.2, \quad b_{3}=-1.75 .
$$

The values of $a_{i}$ and $b_{i}$ are constrained by the condition of reaching the Stefan-Boltzmann limit at $T \rightarrow \infty$ and by imposing the presence of a first-order phase transition at $T_{0}$, which is a further 
parameter of the model. In the absence of dynamical quarks, from lattice calculations one expects a deconfinement temperature $T_{0}=270 \mathrm{MeV}$. However, it has been argued that in the presence of light dynamical quarks this temperature scale should be adequately reduced to about 210 and $190 \mathrm{MeV}$ for the case of two and three flavors, respectively, with an uncertainty of about $30 \mathrm{MeV}$ [33]. Besides the logarithmic function in Eq. (31), other forms for the Polyakov-loop potential can be found in the literature. In the following some of them will be considered, and the effect of the parameter $T_{0}$ on the phase transitions will be analyzed.

\section{B. Thermodynamics}

To investigate the phase transitions and the temperature dependence of thermodynamical quantities within our model, we consider the thermodynamical potential per unit volume at the mean field level. We will proceed by using the standard Matsubara formalism, following the same prescriptions as in previous works, see e.g. Refs. [27, 34]. In this way we obtain

$$
\Omega^{\mathrm{MFA}}=\Omega^{\mathrm{reg}}+\Omega^{\mathrm{free}}+\mathcal{U}(\Phi, T)+\Omega_{0}
$$

where

$$
\begin{aligned}
\Omega^{\mathrm{reg}}= & -2 T \sum_{n=-\infty}^{\infty} \sum_{c, f} \int \frac{d^{3} p}{(2 \pi)^{3}} \log \left[\frac{p_{n c}^{2}+M_{f}^{2}\left(p_{n c}\right)}{Z^{2}\left(p_{n c}\right)\left(p_{n c}^{2}+m_{f}^{2}\right)}\right] \\
& -\left(\bar{\zeta} \bar{R}+\frac{G}{2} \bar{R}^{2}+\frac{H}{4} \bar{S}_{u} \bar{S}_{d} \bar{S}_{s}\right)-\frac{1}{2} \sum_{f}\left(\bar{\sigma}_{f} \bar{S}_{f}+\frac{G}{2} \bar{S}_{f}^{2}\right), \\
\Omega^{\text {free }}= & -2 T \sum_{c, f} \sum_{s= \pm 1} \int \frac{d^{3} p}{(2 \pi)^{3}} \operatorname{Re} \log \left[1+\exp \left(-\frac{\epsilon_{f p}+\imath s \phi_{c}}{T}\right)\right] .
\end{aligned}
$$

Here we have defined $p_{n c}^{2}=\left[(2 n+1) \pi T+\phi_{c}\right]^{2}+\vec{p}^{2}, \epsilon_{f p}=\sqrt{\vec{p}^{2}+m_{f}^{2}}$. The sums over color and flavor indices run over $c=r, g, b$ and $f=u, d, s$, respectively, and the color background fields are $\phi_{r}=-\phi_{g}=\phi_{3}, \phi_{b}=0$. The term $\Omega_{0}$ is just a constant that sets the value of the thermodynamical potential at $T=0$.

Now, from the thermodynamic potential we can calculate various thermodynamic quantities such as the energy and entropy densities, which are given by

$$
\varepsilon=\Omega+T s, \quad s=-\frac{\partial \Omega}{\partial T} .
$$

We are also interested in the behavior of the quark condensates and the corresponding chiral susceptibilities, defined by

$$
\langle\bar{q} q\rangle=\frac{\partial \Omega}{\partial m_{q}}, \quad \chi_{q}=\frac{\partial\langle\bar{q} q\rangle}{\partial m_{q}}=\frac{\partial^{2} \Omega}{\partial m_{q}^{2}}, \quad q=u, d, s .
$$


For large temperatures, the behavior of the regularized quark condensates is dominated by the free contribution, which grows with $T$ as $\langle\bar{q} q\rangle \sim-m_{q} T^{2}$. Therefore, in order to analyze the chiral restoration transition it is normal to define a subtracted chiral condensate

$$
\langle\bar{q} q\rangle_{\mathrm{sub}}=\frac{\langle\bar{u} u\rangle-\frac{m_{u}}{m_{s}}\langle\bar{s} s\rangle}{\langle\bar{u} u\rangle_{0}-\frac{m_{u}}{m_{s}}\langle\bar{s} s\rangle_{0}},
$$

where we have also introduced a normalization factor given by the values of the chiral condensates at zero temperature.

\section{Numerical Results}

We present here our numerical results for the quantities defined in the previous section, considering different form factors and Polyakov loop potentials. Let us start by taking into account the parameterization set II, based on lattice QCD results for the quark propagators, and the logarithmic Polyakov-loop potential in Eq. (31). In Fig. 2 we quote the corresponding behavior of the subtracted chiral condensate, the traced Polyakov loop $\Phi$ and the associated susceptibilities as functions of the temperature. In the upper panel we show the results for the subtracted chiral condensate $\langle\bar{q} q\rangle_{\text {sub }}$ and the traced Polyakov loop $\Phi$, for $T_{0}=270$ and $200 \mathrm{MeV}$ (dashed and solid curves, respectively). As stated, $T_{0}=270 \mathrm{MeV}$ is the deconfinement transition temperature obtained from lattice calculations in pure gauge QCD, while we have taken $T_{0}=200 \mathrm{MeV}$ as a reference temperature arising from the corresponding rescaling in the presence of dynamical quarks [33]. For comparison we also include lattice QCD data taken from Refs. [35, 36]. As expected, it is found that when the temperature is increased the system undergoes both the chiral restoration and deconfinement transitions, which proceed as smooth crossovers for the considered values of $T_{0}$. In the central and lower panels of Fig. 2 we show the curves for the Polyakov loop susceptibility — defined as $d \Phi / d T$ - and the chiral susceptibilities $\chi_{u, s}$ [given by Eq. (37)] as functions of the temperature. As usual, we take the position of the peaks to define the corresponding transition critical temperatures. From the figure it is seen that the curves get steeper for lower values of $T_{0}$; in fact, first order phase transitions are found for $T_{0} \lesssim 185 \mathrm{MeV}$. In addition, in the curves for $\chi_{s}$ it is possible to identify a second, broad peak that allows us to define an approximate critical temperature for the restoration of the full SU(3) chiral symmetry. For clarity we have plotted in the graphs the subtracted susceptibilities $\bar{\chi}_{q} \equiv \chi_{q}-\chi_{q}(T=0)$.

It is seen that both the $\mathrm{SU}(2)$ chiral restoration and deconfinement transitions occur essentially at the same critical temperatures, in agreement with lattice QCD results. The numerical values 


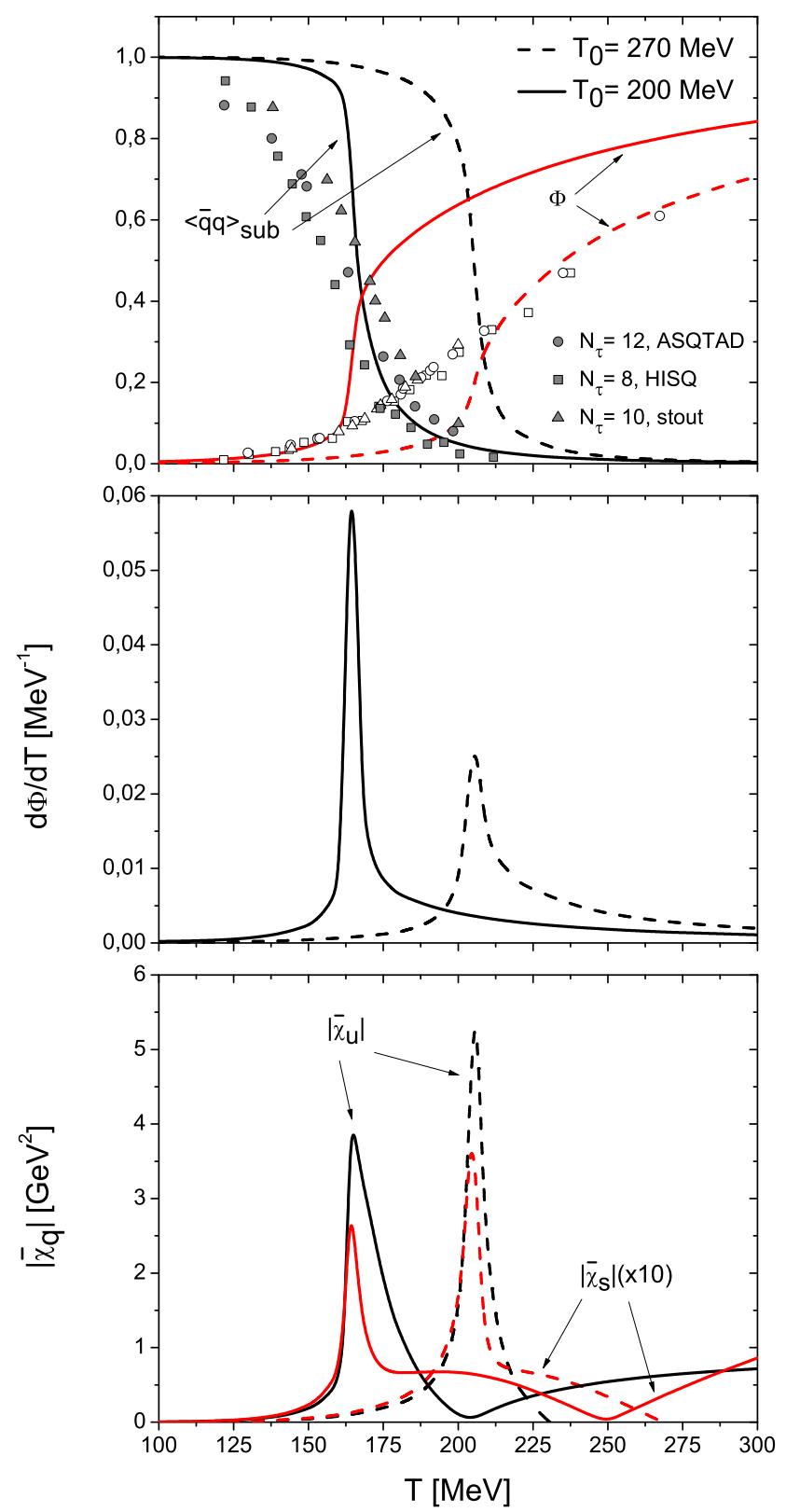

Figure 2: Subtracted chiral condensate, Polyakov loop, chiral susceptibilities, and Polyakov loop susceptibility $d \Phi / d T$ as functions of the temperature. Solid (dashed) curves correspond to parameter set II, for a logarithmic Polyakov loop potential with $T_{0}=200(270) \mathrm{MeV}$. Triangles, circles, and squares stand for lattice QCD results from Refs. [35, 36 ].

from the plots in Fig. 2 are $T_{c} \simeq 200 \mathrm{MeV}$ and $T_{c} \simeq 165 \mathrm{MeV}$ for $T_{0}=270$ and $200 \mathrm{MeV}$, respectively, while lattice $\mathrm{QCD}$ analyses lead to a transition temperature of about $160 \mathrm{MeV}$ [35, 36]. Thus, the agreement with lattice QCD data favors the suggested rescaling of the reference temperature $T_{0}$ from the pure gauge transition temperature towards values around $200 \mathrm{MeV}$.

The above results, which correspond to the lattice QCD-inspired form factors of our parame- 
terization set II, are qualitatively similar to those obtained for the case of set I, based on Gaussian form factors. In order to compare the features of parameterizations I and II, it is useful to consider other thermodynamical quantities, such as the interaction energy and the entropy. The corresponding curves are shown in Fig. 3, where we plot the normalized interaction energy $(\varepsilon-3 p) / T^{4}$ (left) and the normalized entropy density $s / s_{S B}$ (right), where $s_{S B}$ stands for the entropy density Stefan-Boltzmann limit. Dashed and solid curves correspond to parameterization sets I and II, respectively, for the logarithmic Polyakov loop potential in Eq. (31) with $T_{0}=200 \mathrm{MeV}$. We have included, for comparison, three sets of lattice data, taken from Refs. 36 38]. It can be seen that for both the interaction energy and the entropy, the curves for set I show a pronounced dip at about $T \sim 300 \mathrm{MeV}$, which is not observed in the case of set II, where the falloff is smooth. In order to trace the source of this effect we have also considered a third parameterization set III in which the form factor $g(p)$ has a Gaussian shape as in set I, but we do not include the coupling driven by the currents $j^{r}(x)$ [i.e. there is no wave function renormalization, $Z(p)=1$ ]. This parameterization has previously been considered in Ref. [6], where the values of model parameters can be found (see also Ref. [8]). In Fig. 3 it corresponds to the dashed-dotted curve, which does not show the mentioned dip. This indicates that the effect can be attributed to the exponential behavior of the form factor $f(p)$ in the wave function renormalization for set I. Moreover, our results can also be compared with those obtained from the parameterization considered in Ref. [26], where the form factors are introduced so as to fit lattice results for the quark propagator (as in our set II), but $f(p)$ is assumed to have a Gaussian shape. The curves for the interaction energy and the entropy for this model (dotted lines in Fig. 3) are similar to those obtained for our parameterization set I. Thus, from the comparison with lattice data, one can conclude that the choice of a powerlike behavior for $f(p)$, such as that proposed in Eqs. (29-30), turns out to be more adequate than the exponential one.

Another aspect to be analyzed is the steepness of the curves in the transition region. From both the plots in Figs. 2 and 3, it is seen that the transition predicted by the nlPNJL models is too sharp in comparison with lattice estimations. In order to study the robustness of this behavior, it is interesting to consider different forms for the Polyakov loop potential proposed in the literature. Besides the logarithmic form in Eq. (31), a widely used potential is that given by a polynomic function based on a Ginzburg-Landau ansatz [12, 39]:

$$
\frac{\mathcal{U}_{\text {poly }}(\Phi, T)}{T^{4}}=-\frac{b_{2}(T)}{2} \Phi^{2}-\frac{b_{3}}{3} \Phi^{3}+\frac{b_{4}}{4} \Phi^{4}
$$



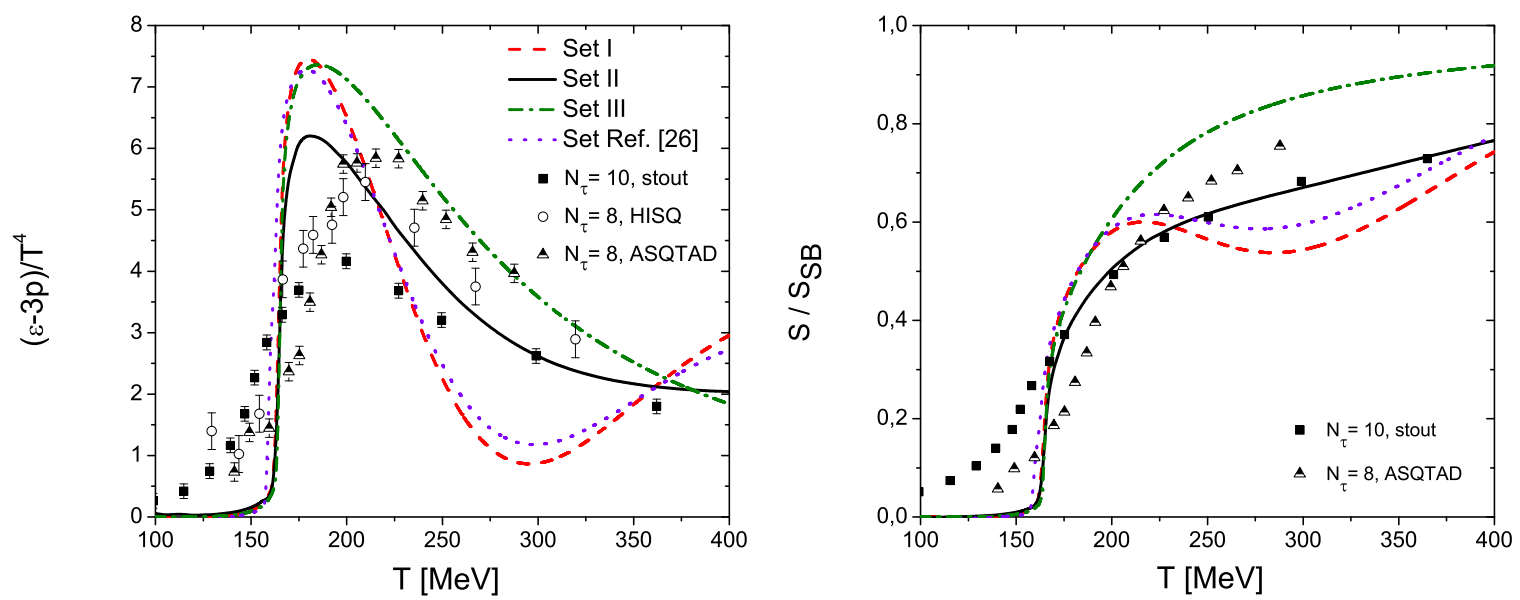

Figure 3: Normalized interaction energy (left) and entropy density (right) as functions of the temperature, for different model parameterizations. Curves correspond to nlPNJL models with logarithmic Polyakov loop potentials, with $T_{0}=200 \mathrm{MeV}$. Squares, circles, and triangles stand for lattice data from Refs. [36], 37] and [38], respectively.

where

$$
b_{2}(T)=a_{0}+a_{1}\left(\frac{T_{0}}{T}\right)+a_{2}\left(\frac{T_{0}}{T}\right)^{2}+a_{3}\left(\frac{T_{0}}{T}\right)^{3} .
$$

Here the reference temperature $T_{0}$ plays the same role as in the logarithmic potential in Eq. (311). Once again, the parameters can be fitted to pure gauge lattice QCD results so as to reproduce the corresponding equation of state and Polyakov loop behavior (numerical values can be found in Ref. [12]). Another widely considered form is the Polyakov loop potential proposed by Fukushima [10, 40], which includes both a logarithmic piece and a quadratic term with a coefficient that falls exponentially with the temperature:

$$
\mathcal{U}_{\text {Fuku }}(\Phi, T)=-b T\left[54 \exp (-a / T) \Phi^{2}+\log \left(1-6 \Phi^{2}+8 \Phi^{3}-3 \Phi^{4}\right)\right] .
$$

Values of dimensionful parameters $a$ and $b$ are given in Ref. [40] (notice that these lead to $T_{c} \simeq 200 \mathrm{MeV}$, a somewhat large transition temperature in comparison with present lattice QCD estimations). Finally, we consider here the "improved" Polyakov loop potential forms recently proposed in Ref. [41], where the full QCD potential $\mathcal{U}_{\text {glue }}$ is related to a Yang-Mills potential $\mathcal{U}_{\text {YM: }}$

$$
\frac{\mathcal{U}_{\text {glue }}\left(\Phi, t_{\text {glue }}\right)}{T^{4}}=\frac{\mathcal{U}_{\mathrm{YM}}\left[\Phi, t_{\mathrm{YM}}\left(t_{\text {glue }}\right)\right]}{T_{\mathrm{YM}}^{4}}
$$

where

$$
t_{\mathrm{YM}}\left(t_{\text {glue }}\right)=0.57 t_{\text {glue }}=0.57\left(\frac{T-T_{c}^{\text {glue }}}{T_{c}^{\text {glue }}}\right) .
$$


The dependence of the potential on the Polyakov loop $\Phi$ is taken from an ansatz such as those in Eq. (39) or (31), while for $T_{c}^{\text {glue }}$ a preferred value of $210 \mathrm{MeV}$ is obtained [41].
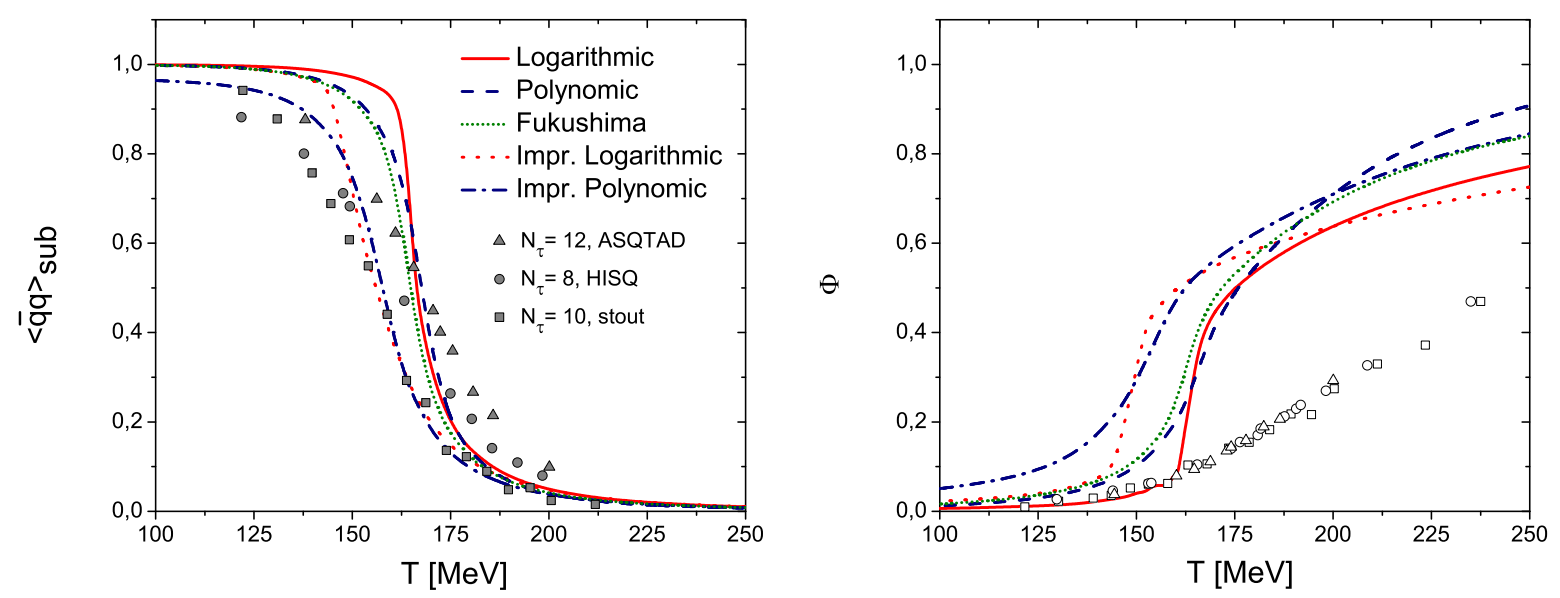

Figure 4: Subtracted chiral condensate (left) and traced Polyakov loop (right) as functions of the temperature, for different Polyakov loop potentials. Curves correspond to parameterization set II. Squares, circles, and triangles stand for lattice data from Refs. [35, 36].

The transition shapes induced by these Polyakov loop potentials within our framework are shown in Fig. 4. We have taken $T_{0}=200 \mathrm{MeV}$ for the logarithmic and polynomic potentials, and the parameters $b$ and $T_{c}^{\text {glue }}$ in Eqs. (41) and (43) have been rescaled to $(145 \mathrm{MeV})^{3}$ and $200 \mathrm{MeV}$, respectively, in order to get critical temperatures of about $165 \mathrm{MeV}$. From the curves for the subtracted chiral condensate (left panel) it is seen that for the case of the polynomic and Fukushima potentials the transition is slightly smoother than for the logarithmic one. Moreover, the improved potentials proposed in Ref. [41] lead to even smoother transitions, showing a reasonable agreement with lattice QCD estimations. On the other hand, by looking at the curves for the Polyakov loop $\Phi$ (right panel in Fig. 4) one finds that the transition is too steep in comparison with lattice data. This is a general feature of Polyakov NJL-like models, both local and nonlocal, and also extends to quark-meson models. In fact, as discussed in Refs. [42 44], the strict comparison between our curves and lattice data for the traced Polyakov loop has to be taken with some care, owing to the difference between the definitions of $\Phi$ in the continuum and on the lattice. One should expect a coincidence in the crossover temperatures, which in general appears to be satisfied in the nlPNJL models for the potentials considered here.

It is important to remark that in nlPNJL models one finds an entanglement between both chiral restoration and deconfinement transitions, in agreement with lattice QCD results. This feature is 
usually not observed in local PNJL models, where both transitions appear to be typically separated by about $20 \mathrm{MeV}$, or even more (see e.g. Refs. [45, 46]). Something similar happens in the region of imaginary chemical potential, where the entanglement between both transitions occurs in a natural way within nonlocal models [24], while in the PNJL model it can be obtained only after e.g. the inclusion of an eight-quark interaction [47]. This discrepancy with lattice QCD results can be cured after the inclusion of an "entangled scalar interaction", in which the effective four-quark coupling is a function of the traced Polyakov loop $\Phi$ [48, 49]. It is also worth noticing that while the local PNJL in general predicts smoother transitions than the nlPNJL, this feature should not be seen as a consequence of the nonlocality. In fact, the enhancement of the steepness arises from the feedback between both chiral restoration and deconfinement transitions. This is supported by the results found in the above-mentioned "entangled" PNJL: by including a $\Phi$-dependent interaction that leads to simultaneous critical temperatures at about $175 \mathrm{MeV}$, the transitions become steeper, just as those obtained in nlPNJL models.

Finally, for completeness we quote in Fig. 5 our results for the behavior of the interaction energy and the entropy and energy densities as functions of the temperature, considering both the logarithmic and the polynomic Polyakov loop potentials, as well as the improved polynomic potential from Ref. 41]. The curves correspond to our parameter set II. It is seen that the improved potential seems to be more compatible with lattice results up to the critical temperature, while at higher temperatures the agreement is better for the usual logarithmic and polynomic potentials. Concerning the steepness of the transitions, it is worth mentioning that the behavior may be softened after the inclusion of mesonic corrections to the Euclidean action, since when the temperature is increased the light mesons should be excited before the quarks [4, 7, 8, 50]. The incorporation of meson fluctuations should not modify the critical temperatures, which for the parameters chosen here are in good agreement with lattice estimations.

\section{SUMMARY AND CONCLUSIONS}

We analyze here the features of three-flavor nlPNJL models that include a wave function renormalization in the effective quark propagators. This represents an extension of previous works that consider two-flavor schemes, and three-flavor models with no quark WFR. In this framework, we obtain a parameterization of the model that reproduces lattice QCD results for the momentum dependence of the effective quark mass and WFR, and at the same time leads to an acceptable phenomenological pattern for particle masses and decay constants in both the scalar and pseudoscalar 

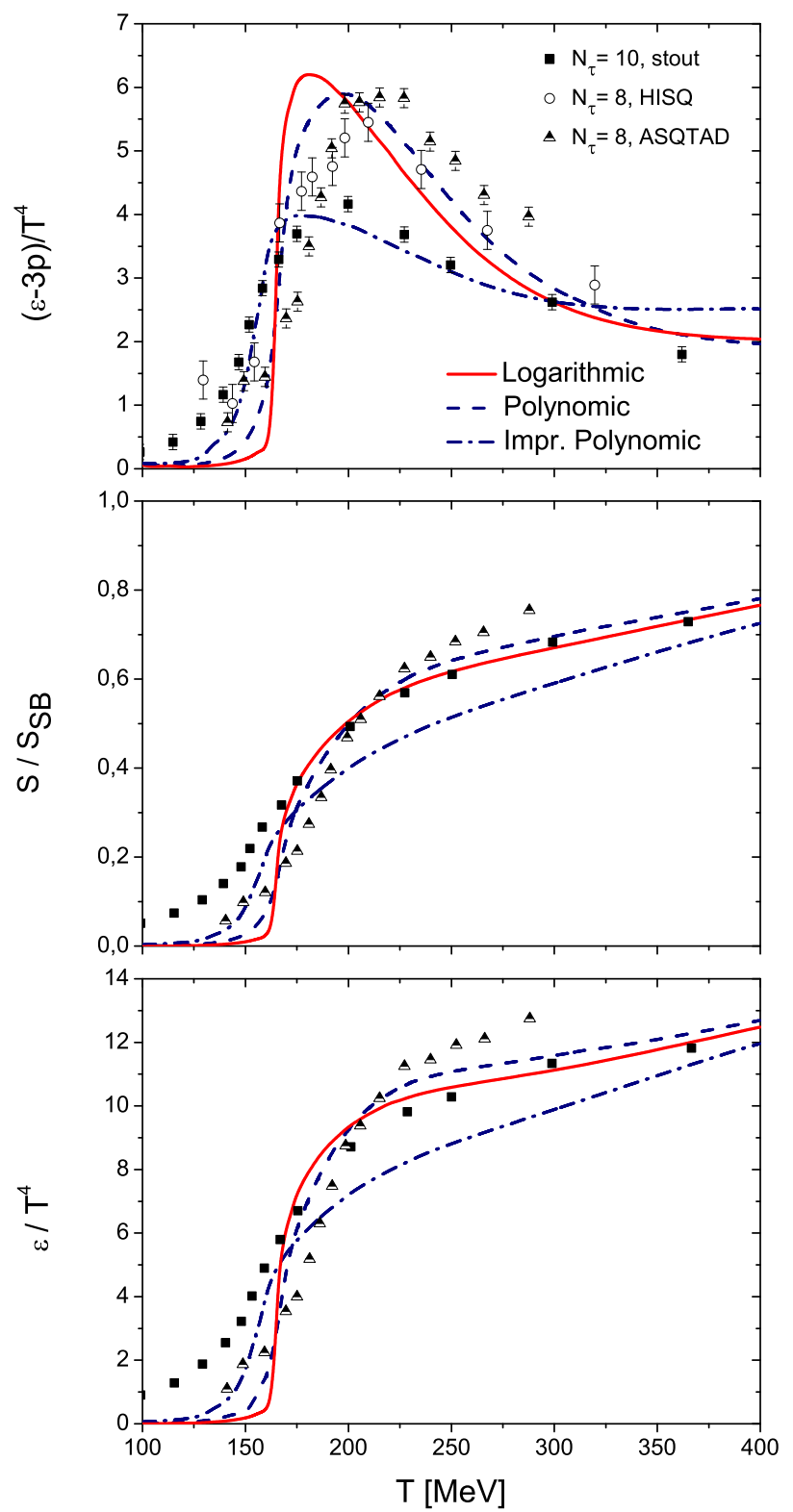

Figure 5: Normalized interaction energy, entropy density and energy density as functions of the temperature, for parameterization set II and three different Polyakov loop potentials. Squares, circles and triangles stand for lattice data from Refs. $[36$ - 38$]$.

meson sectors. For comparison we also consider a parameterization based on Gaussian form factors, which leads to a faster convergence of quark loop integrals. Gaussian and lattice-inspired parameterizations are called here set I and set II, respectively. It is seen that the predictions for meson properties are qualitatively similar in both cases, and they also agree with those obtained previously within three-flavor models with no WFR. 
As a second step we analyze the characteristics of the deconfinement and chiral restoration transitions at finite temperature, introducing the couplings of fermions to a background gauge field and taking the traced Polyakov loop as order parameter for the deconfinement. In general it is found that both transitions occur at the same critical temperature, in agreement with lattice QCD results. This temperature turns out to be strongly dependent on the scale parameter $T_{0}$ in the Polyakov loop potential. A critical temperature of about $170 \mathrm{MeV}$, consistent with that arising from lattice QCD calculations, is obtained for $T_{0} \simeq 200 \mathrm{MeV}$, in agreement with theoretical expectations for a model with two/three light dynamical quarks. On the other hand, in order to distinguish between the different parameterizations and those proposed in related works, we analyze the temperature dependence of the interaction energy and the normalized entropy and energy densities. For these thermodynamical quantities it is seen that the lattice-inspired powerlike parameterization set II shows indeed the best agreement with lattice QCD results, which supports the consistency of our approach. We also consider various possible forms for the Polyakov loop potential, tuning the corresponding parameters so as to obtain critical temperatures in a range compatible with lattice QCD data. It is seen that while the logarithmic potential Eq. (31) leads to rather sharp transitions, for some alternative forms such as the polynomic and Fukushima potentials [Eqs. (39) and (41), respectively] the crossovers tend to be somewhat smoother. Moreover, the improved potentials proposed in Ref. [41] lead to even smoother transitions, showing a reasonable agreement with lattice QCD estimations. The compatibility with the results for the other mentioned thermodynamical quantities is also discussed. Finally, it is worth mentioning that the transitions could also be softened after the incorporation of meson fluctuations, which is presently under study in the context of our models.

\section{Acknowledgments}

This work has been partially funded by CONICET (Argentina) under Grants No. PIP 00682 and No. PIP 02495, and by ANPCyT (Argentina) under Grant No. PICT-2011-0113.

\section{ApPendix: ANALYTIC EXPRESSIONS FOR $G_{M}\left(p^{2}\right)$ FUnCTIONS}

We present here the analytic expressions for the functions $G_{M}\left(p^{2}\right)$ appearing in the quadratic expansion of the Euclidean action, see Eq. (13). Our calculations are in agreement with the results 
reported in Ref. [26]. For the $I \neq 0$ states $\pi, \mathrm{a}_{0}, K$ and $\kappa$ we obtain

$$
\begin{aligned}
& G_{\left(\begin{array}{c}
\pi \\
\mathrm{a}_{0}
\end{array}\right)}(p)=\left(G \pm \frac{H}{2} \bar{S}_{s}\right)^{-1}+4 C_{u u}^{\mp}(p), \\
& G_{\left(\begin{array}{c}
K \\
\kappa
\end{array}\right)}(p)=\left(G \pm \frac{H}{2} \bar{S}_{u}\right)^{-1}+4 C_{u s}^{\mp}(p),
\end{aligned}
$$

where the functions $C_{i j}^{\mp}(p)$, with $i, j=u$ or $s$, are defined as

$$
\begin{aligned}
C_{i j}^{\mp}(p)= & -2 N_{c} \int \frac{d^{4} q}{(2 \pi)^{4}} g^{2}(q) \frac{Z\left(q^{+}\right)}{q^{+2}+M_{i}^{2}\left(q^{+}\right)} \frac{Z\left(q^{-}\right)}{q^{-2}+M_{j}^{2}\left(q^{-}\right)} \\
& \times\left[\left(q^{+} \cdot q^{-}\right) \pm M_{i}\left(q^{+}\right) M_{j}\left(q^{-}\right)\right]
\end{aligned}
$$

with $q^{ \pm}=q \pm p / 2$. In the $I=0$ pseudoscalar sector one has a mixing between the $\eta_{0}$ and $\eta_{8}$ fields. The masses of the physical states $\eta$ and $\eta^{\prime}$ can be obtained from the functions

$$
G_{\left(\begin{array}{c}
\eta \\
\eta^{\prime}
\end{array}\right)}(p)=\frac{G_{88}^{-}(p)+G_{00}^{-}(p)}{2} \mp \sqrt{G_{80}^{-}(p)^{2}+\left(\frac{G_{88}^{-}(p)-G_{00}^{-}(p)}{2}\right)^{2}},
$$

where we use the definitions

$$
\begin{aligned}
G_{00}^{\mp}(p) & =\frac{4}{3}\left[2 C_{u u}^{\mp}(p)+C_{s s}^{\mp}(p)+\frac{6 G \mp H \bar{S}_{s} \pm 4 H \bar{S}_{u}}{8 G^{2}-4 H^{2} \bar{S}_{u}^{2} \mp 4 H G \bar{S}_{s}}\right] \\
G_{88}^{\mp}(p) & =\frac{4}{3}\left[2 C_{s s}^{\mp}(p)+C_{u u}^{\mp}(p)+\frac{6 G \mp 2 H \bar{S}_{s} \mp 4 H \bar{S}_{u}}{8 G^{2}-4 H^{2} \bar{S}_{u}^{2} \mp 4 H G \bar{S}_{s}}\right] \\
G_{80}^{\mp}(p) & =\frac{4}{3} \sqrt{2}\left[C_{u u}^{\mp}(p)-C_{s s}^{\mp}(p) \pm \frac{H\left(\bar{S}_{s}-\bar{S}_{u}\right)}{8 G^{2}-4 H^{2} \bar{S}_{u}^{2} \mp 4 H G \bar{S}_{s}}\right] .
\end{aligned}
$$

The states $\eta$ and $\eta^{\prime}$ are thus defined as

$$
\begin{aligned}
\eta & =\eta_{8} \cos \theta_{\eta}-\eta_{0} \operatorname{sen} \theta_{\eta} \\
\eta^{\prime} & =\eta_{8} \operatorname{sen} \theta_{\eta}^{\prime}+\eta_{0} \cos \theta_{\eta}^{\prime}
\end{aligned}
$$

where the mixing angles $\theta_{\eta}, \theta_{\eta^{\prime}}$ are given by

$$
\tan 2 \theta_{\eta, \eta^{\prime}}=\left.\frac{-2 G_{80}^{-}}{G_{88}^{-}-G_{00}^{-}}\right|_{p^{2}=-m_{\eta, \eta^{\prime}}^{2}}
$$

Finally, for the $I=0$ scalar sector, the quadratic terms involving the fields $\zeta, \sigma_{8}$ and $\sigma_{0}$ are mixed by the $3 \times 3$ matrix

$$
\left(\begin{array}{ccc}
4 C^{\zeta}(p)+G^{-1} & \sqrt{\frac{8}{3}}\left[2 C_{u}^{+\zeta}(p)+C_{s}^{+\zeta}(p)\right] & \frac{4}{\sqrt{3}}\left[C_{u}^{+\zeta}(p)-C_{s}^{+\zeta}(p)\right] \\
\sqrt{\frac{8}{3}}\left[2 C_{u}^{+\zeta}(p)+C_{s}^{+\zeta}(p)\right] & G_{00}^{+}(p) & G_{80}^{+}(p) \\
\frac{4}{\sqrt{3}}\left[C_{u}^{+\zeta}-C_{s}^{+\zeta}(p)\right] & G_{80}^{+}(p) & G_{88}^{+}(p)
\end{array}\right)
$$


where

$$
\begin{aligned}
C^{\zeta}(p)= & \frac{N_{c}}{\kappa^{2}} \int \frac{d^{4} q}{(2 \pi)^{4}} q^{2} f^{2}(q) \sum_{i=1}^{3} \frac{Z\left(q^{+}\right)}{q^{+2}+M_{i}^{2}\left(q^{+}\right)} \frac{Z\left(q^{-}\right)}{q^{-2}+M_{i}^{2}\left(q^{-}\right)} \\
& \times\left[q^{+} q^{-}+\frac{q^{+2} q^{-2}-\left(q^{+} q^{-}\right)^{2}}{2 q^{2}}-M_{i}\left(q^{+}\right) M_{i}\left(q^{-}\right)\right] \\
C_{i}^{+\zeta}(p)= & -\frac{2 N_{c}}{\kappa} \int \frac{d^{4} q}{(2 \pi)^{4}} g(q) f(q) \frac{Z\left(q^{+}\right)}{q^{+2}+M_{i}^{2}\left(q^{+}\right)} \frac{Z\left(q^{-}\right)}{q^{-2}+M_{i}^{2}\left(q^{-}\right)} \\
& \times q \cdot\left[q^{-} M_{i}\left(q^{+}\right)+q^{+} M_{i}\left(q^{-}\right)\right],
\end{aligned}
$$

with $i=u, s$. For a given value of $p^{2}$, we denote the eigenvalues of this matrix by $G_{\zeta}(p), G_{\sigma}(p)$ and $G_{f_{0}}(p)$. As stated in Sec. IID from the functions $G_{\sigma}(p)$ and $G_{f_{0}}(p)$ one can determine the masses of the $\sigma, f_{0}$ physical states [the function $G_{\zeta}(p)$ turns out to be positive definite for the allowed values of $\left.-p^{2}\right]$. The corresponding mixing angles can be obtained in a similar way as in the $\eta$ meson sector, now defining $\mathrm{SO}(3)$ rotation matrices for the $\sigma$ and $f_{0}$ physical states.

[1] C. R. Allton, S. Ejiri, S. J. Hands, O. Kaczmarek, F. Karsch, E. Laermann and C. Schmidt, Phys. Rev. D 68, 014507 (2003); C. R. Allton, M. Doring, S. Ejiri, S. J. Hands, O. Kaczmarek, F. Karsch, E. Laermann and K. Redlich, Phys. Rev. D 71, 054508 (2005).

[2] Z. Fodor and S. D. Katz, JHEP 0404, 050 (2004); Y. Aoki, Z. Fodor, S. D. Katz and K. K. Szabo, JHEP 0601, 089 (2006).

[3] F. Karsch and E. Laermann, in Quark Gluon Plasma III, edited by R.C. Hwa and X. N. Wang (World Scientific, Singapore, 2004), arXiv:hep-lat/0305025.

[4] D. Blaschke, M. Buballa, A. E. Radzhabov and M. K. Volkov, Yad. Fiz. 71, 2012 (2008) [Phys. Atom. Nucl. 71, 1981 (2008)].

[5] G. A. Contrera, D. Gomez Dumm and N. N. Scoccola, Phys. Lett. B 661, 113 (2008).

[6] G. A. Contrera, D. Gomez Dumm and N. N. Scoccola, Phys. Rev. D 81, 054005 (2010).

[7] T. Hell, S. Roessner, M. Cristoforetti and W. Weise, Phys. Rev. D 79, 014022 (2009).

[8] T. Hell, S. Rossner, M. Cristoforetti and W. Weise, Phys. Rev. D 81, 074034 (2010).

[9] P. N. Meisinger and M. C. Ogilvie, Phys. Lett. B 379, 163 (1996).

[10] K. Fukushima, Phys. Lett. B 591, 277 (2004).

[11] E. Megias, E. Ruiz Arriola and L. L. Salcedo, Phys. Rev. D 74, 065005 (2006).

[12] C. Ratti, M. A. Thaler and W. Weise, Phys. Rev. D 73, 014019 (2006).

[13] S. Roessner, C. Ratti and W. Weise, Phys. Rev. D 75, 034007 (2007).

[14] S. Mukherjee, M. G. Mustafa and R. Ray, Phys. Rev. D 75, 094015 (2007).

[15] C. Sasaki, B. Friman and K. Redlich, Phys. Rev. D 75, 074013 (2007).

[16] T. Schafer and E. V. Shuryak, Rev. Mod. Phys. 70, 323 (1998). 
[17] C. D. Roberts and A. G. Williams, Prog. Part. Nucl. Phys. 33, 477 (1994); C. D. Roberts and S. M. Schmidt, Prog. Part. Nucl. Phys. 45, S1 (2000).

[18] S. Noguera and N. N. Scoccola, Phys. Rev. D 78, 114002 (2008).

[19] P. O. Bowman, U. M. Heller, and A. G. Williams, Phys. Rev. D 66, 014505 (2002); P. O. Bowman, U. M. Heller, D. B. Leinweber and A. G. Williams, Nucl. Phys. Proc. Suppl. 119, 323 (2003).

[20] M. B. Parappilly, P. O. Bowman, U. M. Heller, D. B. Leinweber, A. G. Williams and J. B. Zhang, Phys. Rev. D 73, 054504 (2006).

[21] S. Furui and H. Nakajima, Phys. Rev. D 73, 074503 (2006).

[22] K. -I. Kondo, Phys. Rev. D 82, 065024 (2010).

[23] G. A. Contrera, M. Orsaria and N. N. Scoccola, Phys. Rev. D 82, 054026 (2010).

[24] V. Pagura, D. Gomez Dumm and N. N. Scoccola, Phys. Lett. B 707, 76 (2012).

[25] V. Pagura, D. Gomez Dumm and N. N. Scoccola, Phys. Rev. D 87, 014027 (2013).

[26] T. Hell, K. Kashiwa and W. Weise, Phys. Rev. D 83, 114008 (2011).

[27] A. Scarpettini, D. Gomez Dumm and N. N. Scoccola, Phys. Rev. D 69, 114018 (2004).

[28] R. D. Bowler and M. C. Birse, Nucl. Phys. A 582, 655 (1995); R. S. Plant and M. C. Birse, Nucl. Phys. A 628, 607 (1998).

[29] H. Leutwyler, Nucl. Phys. Proc. Suppl. 64, 223 (1998); R. Kaiser and H. Leutwyler, in Non-perturbative Methods in Quantum Field Theory, edited by A.W. Schreiber, A.G. Williams and A.W. Thomas (World Scientific, Singapore, 1998), arXiv:hep-ph/9806336.

[30] T. Feldmann, Int. J. Mod. Phys. A 15, 159 (2000).

[31] J. Beringer et al. [Particle Data Group Collaboration], Phys. Rev. D 86, 010001 (2012).

[32] A. Dumitru, R. D. Pisarski and D. Zschiesche, Phys. Rev. D 72, 065008 (2005).

[33] B. -J. Schaefer, J. M. Pawlowski and J. Wambach, Phys. Rev. D 76 , 074023 (2007); B. -J. Schaefer, M. Wagner and J. Wambach, Phys. Rev. D 81 , 074013 (2010).

[34] D. Gomez Dumm and N. N. Scoccola, Phys. Rev. D 65, 074021 (2002); Phys. Rev. C 72, 014909 (2005).

[35] S. Borsanyi et al. [Wuppertal-Budapest Collaboration], JHEP 1009, 073 (2010).

[36] A. Bazavov et al. [HotQCD Collaboration], J. Phys. Conf. Ser. 230, 012014 (2010).

[37] A. Bazavov et al., Phys. Rev. D 80, 014504 (2009).

[38] S. Borsanyi, G. Endrodi, Z. Fodor, A. Jakovac, S. D. Katz, S. Krieg, C. Ratti and K. K. Szabo, JHEP 1011, 077 (2010).

[39] O. Scavenius, A. Dumitru and J. T. Lenaghan, Phys. Rev. C 66, 034903 (2002).

[40] K. Fukushima, Phys. Rev. D 77, 114028 (2008) [Erratum-ibid. D 78, 039902 (2008)].

[41] L. M. Haas, R. Stiele, J. Braun, J. M. Pawlowski and J. Schaffner-Bielich, Phys. Rev. D 87, 076004 (2013).

[42] J. Braun, H. Gies and J. M. Pawlowski, Phys. Lett. B 684, 262 (2010).

[43] F. Marhauser and J. M. Pawlowski, arXiv:0812.1144 [hep-ph].

[44] T. K. Herbst, M. Mitter, J. M. Pawlowski, B. -J. Schaefer and R. Stiele, arXiv:1308.3621 [hep-ph]. 
[45] P. Costa, M. C. Ruivo, C. A. de Sousa, H. Hansen and W. M. Alberico, Phys. Rev. D 79, 116003 (2009).

[46] W. -j. Fu, Z. Zhang and Y. -x. Liu, Phys. Rev. D 77, 014006 (2008).

[47] Y. Sakai, K. Kashiwa, H. Kouno, M. Matsuzaki and M. Yahiro, Phys. Rev. D 79, 096001 (2009).

[48] Y. Sakai, T. Sasaki, H. Kouno and M. Yahiro, Phys. Rev. D 82, 076003 (2010).

[49] T. Sasaki, Y. Sakai, H. Kouno and M. Yahiro, Phys. Rev. D 84, 091901 (2011).

[50] A. E. Radzhabov, D. Blaschke, M. Buballa and M. K. Volkov, Phys. Rev. D 83, 116004 (2011). 